Nouvelles perspectives en sciences sociales

Revue internationale de systémique complexe et d'études relationnelles

Communication internationale et communication

interculturelle. Regards épistémologiques et espaces de

pratique, Christian Agbobli et Gaby Hsab, Québec, Presses de

l’université du Québec, 2011

\title{
Martin David-Blais
}

Volume 9, numéro 1, novembre 2013

URI : https://id.erudit.org/iderudit/1024048ar

DOI : https://doi.org/10.7202/1024048ar

Aller au sommaire du numéro

\section{Éditeur(s)}

Prise de parole

ISSN

1712-8307 (imprimé)

1918-7475 (numérique)

Découvrir la revue

Citer ce compte rendu

David-Blais, M. (2013). Compte rendu de [Communication internationale et communication interculturelle. Regards épistémologiques et espaces de pratique, Christian Agbobli et Gaby Hsab, Québec, Presses de l'université du Québec, 2011]. Nouvelles perspectives en sciences sociales, 9(1), 347-352. https://doi.org/10.7202/1024048ar d'utilisation que vous pouvez consulter en ligne. 


\section{Comptes rendus de lecture}

\section{Communication internationale et communication interculturelle. Regards épistémologiques et espaces de pratique}

Christian Agbobli et Gaby Hsab, , Québec, Presses de l'université du Québec, 2011.

par Martin David-Blais

Université Saint-Paul, Ottawa

Cet ouvrage collectif est en quelque sorte le résultat d'une opération du Groupe d'études et de recherches sur la communication internationale et la communication interculturelle, le GERACII (de l'UQAM) en vue de se faire connaître. Même si l'objectif de cette opération consiste à mettre en évidence les convictions fondamentales dudit groupe ainsi que la spécificité de sa démarche de recherche, le résultat d'ensemble prend une allure disparate. Cela ressemble beaucoup à ces actes de colloque où chaque participant $\mathrm{y}$ va d'une contribution personnelle faisant part de son travail du moment.

Bien que l'ouvrage comporte trois parties, il se trouve fondamentalement divisé en deux composantes : 1) les textes théoriques et 2) les études de cas (parties 2 et 3). Disons pour être plus précis que la première partie est de l'ordre de la discussion " méta ", c'est-à-dire qu'elle est faite de textes consacrés soit à la description et à la délimitation des deux champs en question, la 
communication internationale et la communication interculturelle (on procède à une sorte de cartographie des champs, lesquels se trouvent divisés en un certain nombre de paradigmes), soit à la présentation des grandes options théoriques et méthodologiques du groupe, soit encore à des considérations épistémologiques. Les six derniers articles du livre sont des études de cas que les responsables du collectif classifient sur la base des catégories " communication internationale » et " communication interculturelle ». Toutefois, après lecture des articles, on ne voit pas très bien ce qui distingue les deux catégories. En fait, le partage des textes des parties 2 et 3 semble finalement reposer sur un principe classificatoire très simple : les articles consacrés à l'immigration au Québec (trois articles dont deux consacrés à la crise des accommodements raisonnables) et les autres qui ne se ressemblent guère les uns par rapport aux autres).

Le sous-titre du livre (regards épistémologiques et espaces de pratique) est à mon sens quelque peu trompeur. Il donne explicitement à penser, comme on vient de le voir, qu'il est à la fois question de pratiques et d'épistémologie. Or, quelqu'un qui voudrait réfléchir à la pratique de la communication "inter » (internationale ou interculturelle) ne trouvera pas grand-chose à se mettre sous la dent. Traiter de pratique au sens fort, c'est discuter de diagnostic, de mode de relation, de stratégies d'intervention et d'actes précis (en isolant les conditions dans lesquels on peut les exercer). Rien de tout cela dans le présent livre. Je ne vois pas en quoi cet ouvrage pourrait être utile, par exemple, à des responsables d'un quelconque ministère qui chercheraient à travailler étroitement avec des populations montagnaise et atikamekw parce qu'ils désirent prendre au sérieux les prétentions de ces nations quant à la différence - très grande - de leurs cultures respectives par rapport au mode de pensée blanc et occidental. Il en va de même pour des enseignants, des travailleurs sociaux, etc., à qui l'on demanderait de travailler plus adéquatement auprès de clientèles culturellement disparates : ce livre ne leur sera pas d'une grande aide, ni du point de vue pratique, ni du point de vue praxéologique. La portée épistémo- 
logique du livre n'est guère plus satisfaisante. Premièrement, l'analyse des implications de la démarche que l'on préconise demeure très en surface. Voilà plus de 150 ans que la tradition compréhensive (issue de l'herméneutique des philosophes et des philologues) a pignon sur rue au sein des sciences sociales et que l'on fait grand cas de l'expérience et de la production de sens en contexte d'action. Un lecteur qui a quelque peu lu sur Weber, Mead, l'école de Chicago, Schütz, l'interactionnisme symbolique, etc., ne fera aucune découverte. Deuxièmement, le travail de comparaison inter-paradigmatique demeure dans l'ensemble trop sommaire pour être convaincant. Un exemple : je veux bien supposer pour les fins de la discussion qu'il existe deux grandes conceptions de la communication, l'une est télégraphique et l'autre est axées sur les rituels (référence à Carey), mais encore faudrait-il qu'on nous montre en quoi ces conceptions sont au coeur de l'organisation des discours analytiques et qu'il y a là deux matrices conceptuelles dont on voit qu'elles sont récurrentes et indépassables. On s'en tient à une dichotomisation assez gratuite - qui évoque le partage classique que l'on faisait encore récemment entre la communication télégraphique et la communication orchestrale (je pense à Winkin à Lohisse alors qu'ils ont cherché à diffuser les travaux de l'école de Palo Alto). Autre exemple : il me semble quelque peu abusif de soutenir que toute démarche de nature positiviste n'a rien à dire sur l'expérience subjective du réel social ou de la culture, et ce, parce que le positivisme aborde les phénomènes de l'extérieur, sans tenir compte du sens ressenti, construit, exprimé. La psychologie sociale, qui travaille soit de manière expérimentale, soit au moyen d'outils statistiques, est une illustration éclatante du contraire puisqu'elle a développé les notions d'attribution, de représentation, de " dissonance cognitive », de biais, etc.

Malgré ces réserves importantes, le livre est intéressant en ce qu'il apporte une assez bonne idée du type de travail qui peut se faire au sein et autour du GERACII.

Revenons à la première partie. Je ne puis tout présenter, les cinq textes qu'on y trouve étant assez différents. Ce qui frappe le 
plus néanmoins, c'est qu'on y exprime avec force un certain nombre de choix fondamentaux, lesquels semblent faire passablement consensus dans la mouvance du groupe. Je me réfère surtout ici au texte de Hsab et Stoicu et à celui d'Agbobli. Le premier de ces choix concerne l'importance de l'expérience et du sens dans l'analyse des phénomènes de culture et de relation entre groupes nationaux. Le GERACII se positionne donc, on l'aura compris, du côté de la sociologie compréhensive par opposition à toutes les démarches plutôt objectivistes. Le second choix porte sur l'importance du phénomène de rencontre (rencontre des nations, rencontre des cultures), ce qui conduit l'analyse à considérer les réactions - cognitives, émotives, réflexives - des populations et des individus qui les composent de même que les enjeux - identités, places, pouvoir - que ces rencontres font surgir. À l'évidence, le GERACII se range aussi dans le camp interactionniste. De ces deux choix découlent diverses conséquences. D'abord, il y a l'importance du « construit en situation " : pour les théoriciens de ce collectif, les comportements, les attitudes, les représentations, les discours et même les institutions doivent être considérés comme des produits sociaux et historiques tributaires des interactions entre groupes. Autre conséquence de ces deux choix de fond : on affiche une nette préférence pour les méthodologies de type herméneutique par opposition aux démarches plus positivistes de même qu'aux démarches de facture holiste. Tout cela est très clairement exprimé. Par contre, le propos de la première partie ne va pas beaucoup plus loin; l'on a surtout droit à des efforts de délimitation et description des deux champs en question. C'est pourquoi, après lecture, on continue à se poser d'innombrables questions, dont certaines sont très basiques. Par exemple, on n'y trouve pas dans le livre une conceptualisation précise de ce qu'est la culture et de son statut dans l'analyse des phénomènes interculturels. Il en va de même pour le concept de nation. Mais il reste que l'on trouve dans cet ouvrage les fondements d'un programme de travail, ce qui donne envie de voir la suite.

Je relève un troisième grand choix qui, lui, est susceptible de 
poser problème : l'idée d' "inter " est ici très circonscrite. On s'en tient à la réalité des nations au sens large et des flux migratoires. Ce faisant, l'objet d'étude par excellence est l'expérience de l'immigration et l'accueil (problématique) de l'immigrant. Voilà qui apporte une certaine simplicité opératoire, mais on pourra objecter que le culturel et l'interculturel peuvent très bien être conçus du point de vue du genre ou de la religion, par exemple. Cette question n'est aucunement envisagée.

Un mot maintenant sur les études de cas. La lecture des articles qui composent les parties 2 et 3 est très intéressante si l'on considère chaque texte séparément. Ils sont tous bien faits et suscitent le désir d'en savoir davantage sur les travaux dont ils rendent compte. Par contre, l'ensemble n'a pas beaucoup d'unité, ce qui ne correspond guère à l'intention générale de l'opération collective du GERACII. Qui plus est, aucun de ces textes ne me semble conçu en relation étroite avec les considérations théoriques de la première partie. Prenons par exemple le texte de Jean-Jacques Maomra Bogui et Myriam Montagut-Lobjoit consacré à la production et la réception de la nouvelle en Côte d'Ivoire dans le contexte de la relation à la France. Le texte est fort sérieux et fait état d'un vaste travail de recherche; seulement, la démarche des auteurs ne fait aucune place à l'expérience intime et subjective de l'altérité dont il a été dit qu'elle doit être au cœur de l'analyse. En fait, on a affaire à un travail classique en études des médias, la référence théorique première des auteurs étant l'agenda setting.

La troisième partie comporte des textes sur la réalité de l'immigration au Québec, dont deux traitent de la crise dite des accommodements raisonnables. À nouveau, ce sont des travaux sérieux qui pourraient être présentés dans des colloques de communication. Et à nouveau, je constate l'absence de lien avec la réflexion de Hsab, Stoicu ou Agbobli présentée en première partie. Pourtant, la question des accommodements raisonnables (Chahid et Potvin parlent d'une crise) constituait un fort bon objet pour qui s'intéresse à l'expérience de l'autre en contexte d'interaction entre communautés. Les deux textes présentés 
procèdent de stratégies analytiques évacuant complètement la prise en compte de l'expérience et du sens en contexte concret d'action. Dans un cas, l'auteur diagnostique une grande peur collective et fait alors intervenir un phénomène mythique qui provoque alors une prolifération d'interventions hautement idéologiques sans relation avec la réalité. Dans l'autre, l'auteure s'intéresse au discours médiatique (propos de commentateurs et avis de leurs lecteurs) pour mettre en évidence les nombreuses distorsions qu'ils comportent et la prolifération de procédés rhétoriques mis au service d'un point de vie essentiellement raciste. Ce qui m'ennuie ici, ce n'est pas la nature de ces stratégies pour elles-mêmes, elles sont classiques et légitimes; ce qui fait problème de mon point de vue, c'est le hiatus avec ce qui est annoncé en première partie. Il y a absence de considération pour le sens et l'expérience de celles et ceux qui se sont inquiétés de la portée des accommodements raisonnables : de l'extérieur, on les juge peu ou prou délirants, racistes ou les deux à la fois. Voilà qui entre en contradiction complète avec l'un des principes cardinaux de la tradition compréhensive en sciences humaines, à savoir la mise en suspens par l'analyste de ses présupposés normatifs (bracketing). 